\title{
CUIDAR LA INFANCIA: ASEGURAR LA RAZA
}

Cuidar da infância: proteger a raça

To care for children: to ensure the race

\section{Martín Caldeiro}

Instituto Superior de Educación Física (ISEF), Montevideo, Uruguay. Telf.: 099966

\section{Correo electrónico: martincaldeiro@gmail.com}

\section{Resumen}

Este artículo toma como referencia mi tesis de maestría en la que analizo los sentidos que adquirieron los discursos sobre el juego en el marco de la educación escolar y particularmente en la Educación Física en las primeras tres décadas del siglo XX en Uruguay. Abordé la cuestión de la infancia en tanto sujeto histórico moderno, asociado a procesos de producción disciplinarios y bio-políticos, que conformaron un escenario en el cual quedó inscripto el cuerpo de la infancia. Contexto en el que emergieron prácticas que recepcionaron discursos de carácter eugenésicos, solidarios con fines de protección y mejora de la raza, teniendo en la higiene, preocupada por la salud del cuerpo del individuo y de la población, un canal para su difusión. Fomentar el "crecimiento y desarrollo normal del organismo" (CNEF, 1923: 11) ${ }^{1}$ se tornaba un objetivo de la educación física, con efectos sobre la sociedad toda. La herencia europea definía los caracteres y cualidades de la descendencia en el nuevo país moderno, pero no sin las propias modificaciones, producto de las "mezclas de nacionalidades y razas [...] dando origen a lo que podríamos llamar, una nueva nacionalidad con caracteres propios" (CNEF, 1923: $9-10)^{2}$.

Recupero documentos de la Comisión Nacional de Educación Física y una conferencia de Alejandro Lamas con patrocinio de la CNEF, en los que se presentan diferentes estrategias de gobierno del cuerpo, a saber: campamentos, plan de educación física infantil y el juego, con el fin del mejoramiento de la raza.

Palabras Clave: infancia, eugenismo, raza, biopolítica, Educación Física

\footnotetext{
${ }^{1}$ El destacado es mío

${ }^{2}$ El destacado es mío
} 


\begin{abstract}
This article takes as reference my master's thesis in which I analyze the senses that acquired the discourses about the game in the framework of school education and particularly in Physical Education in the first three decades of the twentieth century in Uruguay.

Address the question of childhood as a modern historical subject, associated with processes of disciplinary and biopolitic production, which forms a scenario in which the body of childhood is inscribed. Context in which discourses and practices emerged that received solidarity discourses for the purpose of protection and improvement of the race, taking hygiene, concerned about the health of the body of the individual and the population a channel for dissemination. Promote the "growth and normal development of the organismo" (CNEF, 1923: 11) $)^{3}$ became an objective of physical education, with effects on the whole society. The European heritage defined the characters and qualities of the offspring in the new modern country, but not without the own modifications, "due to the mixtures of nationalities and races. giving rise to what we could call a new nationality with its own characteristics" (CNEF, 1923: $9-10)^{4}$.

Recover documents from the National Commission of Physical Education and a conference of Alejandro Lamas with sponsorship of the CNEF, in which different strategies of governing the body are presented, camps plan of physical education for children and play, in order to improve of the race.
\end{abstract}

Keywords: childhood, eugenics, race, biopolitics, Physical Education

\title{
Resumo
}

Este artigo é um recorte de minha tese de mestrado, na qual analiso os sentidos que adquiriram os discursos sobre o jogo no âmbito da educação escolar e particularmente a Educação Física nas primeiras três décadas do século XX no Uruguai. Abordei a questão da infância como um sujeito histórico moderno, associado a processos de produção disciplinares e biopolíticos, que configuraram um cenário no qual se inscreve o corpo da infância. Contexto em que emergiram práticas permeadas de discursos de

\footnotetext{
${ }^{3}$ El destacado y la traducción son mías.

${ }^{4}$ El destacado y la traducción son mías.
} 
caráter eugênico, solidários com fins de proteção e melhoramento da raça, tendo a higiene, esta preocupada com a saúde do corpo do indivíduo e da população, um canal para sua difusão. Fomentar o "crecimiento y desarrollo normal del organismo" (CNEF, 1923:11) se tornava um objetivo da educação física, com efeitos sobre toda a sociedade. A herança europeia definiu os caracteres e qualidades dos descendentes no novo país moderno, mas não sem suas próprias modificações, produto das "mezclas de nacionalidades y razas [...] dando origen a lo que podríamos llamar, una nueva nacionalidad con caracteres propios" (CNEF, 1923: 9 -10). Recupero documentos da Comissão Nacional de Educação Física e uma conferência de Alejandro Lamas, patrocinada pela $\mathrm{CNEF}$, nos quais se apresentam diferentes estratégias de governo do corpo, a saber: acampamentos, planos de educação física infantil e jogo, com o fim de melhoramento da raça.

Palavras Chave: infância, eugenismo, raça, biopolítica, Educação Física

\section{Introducción}

El empirismo o naturismo si se prefiere esta palabra, y el artificialismo, si es permitido tal término, han tenido, tienen y tendrán sus ardientes y sinceros partidarios, especialmente en referencia al cuerpo; por mi parte creo firmemente que cuanto hace el horticultor puede también hacerlo el puericultor y que mejores frutos se cosechan usando del método. - Pero, eso sí, ha de ser realmente cientifico, entendiendo por ello, basado en las leyes biológicas y en la psicología y no apartándose de la naturaleza sino en cuanto se vea perjudicial o inadaptable al medio artificial de vida que la sociedad hace, pues no hay que

olvidar que fuera de la naturaleza vivimos todos y en todo (Lamas, 1912: 33 - 34).

Es posible analizar la recepción del discurso eugenésico ${ }^{5}$ en el Uruguay a través de las políticas educativas desenvueltas a comienzos del siglo XX. Este período es reconocido por varios historiadores: Caetano (2011), Barrán (2009), Nahum (2011) como la época batllista $^{6}$, en la que se forja el país moderno, en consecuencia el ciudadano modelo, respondiendo a la preocupación generalizada de las clases dirigentes, de crear, a partir

\footnotetext{
${ }^{5}$ La Eugenesia es la teoría que pugna por combatir la degeneración de la raza regida por los principio que rigen la biología (Barrán, 1995: 206). Correspondió al inglés Francis Galton (1822-1911), primo de Charles Darwin, impulsar la idea de eugenesia en el mundo a partir de 1883. La definió como la ciencia que procuraría que cada especie, clase o raza, llegase al máximo de sus posibilidades mediante el cruzamiento entre los mejores y la esterilización de los seres biológica y mentalmente defectuosos. Su teoría descansaba sobre dos supuestos, la selección natural darwiniana y la herencia de los caracteres adquiridos (Barrán, 1999: 98).

${ }^{6}$ Entre 1903 - 1907 y en un segundo mandato 1911 - 1915 Batle es presidente del Uruguay.
} 
de una población heterogénea, una nueva y purificada homogeneidad sobre la cual erigir la nacionalidad. A decir de Barrán (1995), la sociedad uruguaya secularizaba su mentalidad, al tiempo que se producían numerosas transformaciones a nivel: económico, político, social y cultural respecto del siglo anterior. En este contexto se crea la Comisión Nacional de Educación Física $(\mathrm{CNEF})^{7}$ en 1911 y en 1923 se formula un Plan de acción ${ }^{8}$ de alcance nacional, publicado por dicha institución, en el que se plantean todas las políticas a ser desenvueltas en el territorio uruguayo, sobre la Educación Física. Moldear al ciudadano moderno era la premisa del gobierno, ¿cuál sería el sujeto que el poder produciría mejor y más rápido? La respuesta no se hace esperar, la infancia ${ }^{9}$. Las políticas implementadas en el marco de la CNEF tuvieron una afanosa preocupación por la infancia, de esta forma se aseguraría la incorporación al proceso civilizatorio a ese nuevo sujeto, asegurando el porvenir del país.

El Plan de acción de 1923, se organizó con discursos y prácticas que se asociaron y especializaron en la producción de la infancia remitiendo de manera directa a la cuestión del cuidado por la degeneración de la raza ${ }^{10}$. Barrán (1995), destaca como una de las características del eugenismo uruguayo, la preferencia por el término especie al

\footnotetext{
${ }^{7}$ Smith se torna el primer presidente de la Comisión Nacional de Educación Física, designado por José Batlle y Ordóñez, Presidente de la República, el 28 de julio de 1911. En julio de 1912, Smith presenta el proyecto de creación de las plazas vecinales de cultura física, que fuera aprobado por la Comisión en agosto del mismo año (RODRÍGUEZ GIMÉNEZ, 2014, p. 138). Al justificar la razón de la creación de las plazas de barrio, decía que el propósito era "perfeccionar la raza humana física y moralmente" (SMITH, 1913, p. 6). Por otro lado, Jess T. Hopkins se radicó en Uruguay en 1912, para encargarse del Departamento de Educación Física, fundado en junio de 1912. En 1913, fue designado Director General de Plazas Vecinales y luego Director Técnico de la CNEF, desde donde impulsó el establecimiento de plazas de deporte (Dogliotti, 2012: 171). A su vez, Hopkins, al referirse a la educación física afirmaba: "toda educación, y especialmente la educación física debe estar basada en un estudio biológico y etnológico de la raza humana" (Dogliotti, 2012: 171).

${ }^{8}$ Fue entregado por una comisión especial, designada del seno de la CNEF, al Ministerio de Instrucción Pública. Este documento constituyó el plan a ser desenvuelto en el campo de la Educación Física en Uruguay conforme al artículo 60 de la Ley de Presupuesto General de Gastos (CNEF, 1923).

9 Los sistemas pedagógicos como la escuela son efecto de la emergencia del nuevo sujeto moderno denominado infancia. Así, un nuevo campo de intervención gubernamental es centro de una gran variedad de discursos, prácticas e instituciones, que se asumieron como responsables por el control de la infancia. En palabras de Rodríguez Giménez, "las sociedades modernas descansan, en gran parte, en la existencia de sistemas educativos nacionales" (Rodríguez Giménez, 2012: 51), que tuvieron en la infancia el sujeto de sus preocupaciones. Concordamos con el autor que no es redundante afirmar que se trató de una invención de la modernidad, y que a infancia no estaba presente hasta el siglo XIII, cuando aparecen imágenes de niños más o menos próximas de las que conocemos contemporáneamente. Para Ariès (1960) el descubrimiento de la infancia propiamente dicha, como categoría social, comienza en el siglo XVIII.

10 "La idea de la degeneración de la «raza» implicaba casi siempre el principio de la transmisión hereditaria de los caracteres patológicos adquiridos, y fue expuesta por vez primera por el médico francés B.A. Morel en 1857, atemorizado por el espectáculo de la degeneración física y «moral» del proletariado de su país" (Barrán, 1999: 36).
} 
de raza (Barrán, 1995: 216). A diferencia de lo que plantea el historiador uruguayo, en los documentos analizados para este artículo $^{11}$, el concepto de raza es notorio ${ }^{12}$. Pero lo importante es destacar la evidencia del discurso eugenésico en el Uruguay, indicada, tal como lo plantea el citado autor, en "la documentación reunida [lo cual] testimonia que el eugenismo o sus ideas claves lograron muy importante difusión e influencia en el Uruguay desde 1900 a 1940 [y] En los años veinte y treinta [...] el eugenismo influyó en la educación estatal" (Barrán, 1995: 223 - 224. El paréntesis es nuestro). Esto nos habilita afirmar, o por lo menos a manejar la hipótesis de una presencia de las ideas eugenistas en el discurso de la Educación Física a comienzos del siglo XX en nuestro país, promovidas a través de diferentes prácticas. Los trabajos de Dogliotti (2012) ${ }^{13}$ y Rodríguez Giménez (2012) ${ }^{14}$, dan cuenta de esta presencia a la que nos referimos. Indicios de esto encontré en mi trabajo de maestría (Caldeiro, 2017), que tomo como referencia para este artículo. Allí pude identificar: -discursos que dan cuenta de la relación que la Educación Física establece con la ciencia, particularmente la ciencia natural (la biología), se explicitaba de la siguiente manera "ella [la Educación Física] es una ciencia [...] basada en las ciencias biológicas y psicológica", y que, "para aplicarla, primero se diagnostica y después se receta" (CNEF, 1923: 9); y -prácticas, teniendo el juego un lugar destacado, asociado a los conceptos de infancia, moral, raza y sexualidad. William en el libro Cultura y Materialismo plantea que la biología tiene en si misma "un fuerte componente social" (Williams, 2012: 111), "la aplicación [...] de la idea biológica al pensamiento social, viene no tanto de Darwin" (Williams, 2012: 112)

11 Los documentos analizados en este trabajo son: la conferencia ofrecida por Lamas, denominada Educación física e intelectual conexas. Un plan de educación física infantil, realizada en el Ateneo de Montevideo el 4 de noviembre de 1911, y publicada con patrocinio de la CNEF; el Plan de Acción de la CNEF de 1923 y el informe del año 1930 titulado Educación Física en el Uruguay, escrito por Julio J. Rodríguez director técnico de la CNEF entre 1920 e 1960.

${ }^{12}$ Habría que rastrear más en profundidad este concepto en el marco de la Educación Física del momento teniendo en cuenta lo que plantea Williams 2012, sobre la palabra raza, la cual fue usada en algunas oportunidades por Darwin "como término biológico para referirse a las especies" (Williams, 2012: 118).

13 “Jess Hopkins y su discípulo J. Rodríguez, a través de sus discursos sobre el cuerpo y la educación física mostraban una interpretación del evolucionismo darwiniano tendiente a la degenerescencia (Vigarello, 2005). Esto implicaba una tergiversación de la teoría de Darwin, en tanto la evolución era vista como un proceso de anti evolución normativa, como un mecanismo de "degeneración" provocada no por causas biológicas de organización interna de los cuerpos sino como consecuencia de los fenómenos sociales del industrialismo" (Dogliotti, 2012: 304).

14 Según este autor la creación de la CNEF, es un acontecimiento altamente significativo en lo que respecta a la triada Estado-educación-población, en el marco de un "programa eugenésico" según Rodríguez Giménez (2012) "El blanco de la intervención estaría definidamente en el cuerpo orgánico, o directamente en el organismo, tanto en su forma individual como colectiva (población), y todas las formas intermedias (familia, escuela, club, etc.)" (2012, p. 179). 
sino de la tradición evolutiva, anterior a Charles Darwin, por lo menos desde finales del siglo XVIII. Consolidándose como sistema de pensamiento a mediados de siglo XIX. "La explicación de los medios de evolución hubo de esperar nuevos descubrimientos, pero la idea ya estaba allí” (Williams, 2012: 112). Spencer, filósofo social acuñó la frase "la supervivencia del más apto", quien creía en "un principio de selección social operativo en la historia humana, [siendo] extremadamente importante que los hombres no interfieran en él, y en especial que los gobiernos no interfieran en él" (Williams, 2012: 112 - 113), oponiéndose a que las clases pobres fueran ayudadas por el estado, ya que de esta forma se preservaría a los "miembros más débiles y menos exitosos de la raza" (Williams, 2012: 113). En definitiva, más allá de la ética de esta posición, no había justificación de una intervención artificial con el fin de preservar a los menos aptos o más débiles, ya que el mismo proceso de selección social estaba creando los tipos más vigorosos (Williams, 2012: 113). A decir de este autor, la idea de que el carácter estaba determinado por el entorno, es central en la teoría del darwinismo social. Robert Owen ${ }^{15}$, continúa explicando el autor, sostenía que se podía reformar el carácter moral de toda la población en un lapso relativamente breve de tiempo, con solo modificar su entorno (Williams, 2012: 113 - 114). Otro aspecto relevante de la visión de Darwin es la idea que "una sociedad que no seleccionaba y descartaba estaba en cierto peligro" (Williams, 2012: 114). En definitiva, si se preserva artificialmente, a aquellos menos dotados o más débiles, la condición general de la raza tiende a deteriorarse, desconociéndose la selección social, determinada por el principio de la supervivencia del más apto. La aplicación de este principio adoptó diferentes variantes, una de ellas fue la eugenesia, que buscó "la reproducción de los tipos mejor dotados", creencia en la "herencia física selectiva" (Williams, 2012: 118). Se pueden identificar dos vertientes de la eugenesia, la positiva: que alienta la reproducción de los más aptos, tanto física como intelectualmente; y la negativa, impedir que los no aptos se reproduzcan. La idea de una raza humana superior, particularmente la anglosajona, encuentra asidero en esta teoría, como la heredera de un derecho natural para imponerse al resto, casi como un imperativo para con la humanidad. ¿Cuál fue la recepción de estos discursos y prácticas por parte de la Educación Física de la época? Los documentos analizados, en este

\footnotetext{
15 Pensador inglés, socialista, promovió el cooperativismo. En 1829 crea la Asociación Británica para la Promoción de la Ciencia Cooperativa.
} 
trabajo, dan cuenta de indicios que permiten pensar una influencia del eugenismo, coincidentemente, los conceptos de raza, herencia, organismo y degeneración, aparecen en los documentos analizados en este trabajo, período en el que, como destacó Barrán (1995) circulan en Uruguay los discursos de la perspectiva eugenésica, así como también una clara y explícita preocupación por el cuidado de la infancia, siendo el higienismo el movimiento determinante.

Patrocinada por la CNEF, Lamas ${ }^{16}$ expone una conferencia en 1911 dedicando un extenso pasaje al juego, justificando, a partir de datos estadísticos obtenidos por el examen individual realizado en las escuelas públicas en el período comprehendido entre noviembre de 1909 y mayo de 1911, la importancia de la Educación Física y "el porvenir de la población futura de la República” (Lamas, 1912: 29). Los números mostraban "un porcentaje de taras hereditarias, de organismos con capitales biológicos pobrísimos [y] con afecciones predisponentes" (Lamas, 1912: 29). Esas notas, a decir de Lamas, indicaban "inferioridad constitucional” y "pobreza orgánica", justificando la intervención: "la vigorización del organismo se impone, pues; -y hay que hacerla empezando por el niño, continuándola en el adulto y prosiguiéndola en el hombre maduro; es condición de existencia" (Lamas, 1912: 32). Surgen así una serie de estrategias educativas: "parques de juegos, las escuelas al aire libre, las copas de leche, la obligatoriedad de la gimnasia científica” (Lamas, 1912: 32). Se puede identificar en el discurso de Lamas, un orden, una serie de etapas sucesivas por las que pasaría un organismo en desarrollo, a saber: niñez, adultez y hombre maduro. En este orden de evolución es que debe hacerse la intervención sobre los cuerpos de los individuos, justificado en el conocimiento de la estadística de ese momento, que enunciaba elevados porcentajes de deterioro orgánico: “organismos con capitales biológicos pobrísimos", “inferioridad constitucional” y "pobreza orgánica", producto de una mala herencia, o sea "taras hereditarias". Curiosamente los discursos sobre la Educación Física

\footnotetext{
${ }^{16}$ Alejandro Lamas fue dentista, Profesor de Educación Física e ganador del primer concurso para formar docentes para el dictado de las primeras aulas "sistemáticas de 'gimnasia' y juegos a partir del año 1905". A comienzos de 1905 fue nominado profesor de Gimnasia de las escuelas de Montevideo. A partir del año 1908 se encargó del dictado de aulas de "gimnasia" en los institutos magistrales, tarea que desenvolvió por poco tiempo. En 1909 fue el principal conferencista de la 2a Conferencia Pedagógica, Una lección de gimnasia. Su valor fisiológico, higiénico y pedagógico, en la que expuso sus ideas a partir de 16 lecciones de gimnasia que conformaban el programa propuesto por él para las escuelas (Dogliotti, 2012: 95-96).
} 
evidencian texturas que se pueden encontrar en las teorías del darwinismo social y de cierto eugenismo, el corte biológico que se hace del cuerpo, la idea del cuerpo organismo que lo esencializa, la preocupación por el deterioro orgánico, las cuestiones de la herencia, la evolución en etapas del cuerpo-organismo, son algunas cosas que aparecen en los discursos presentados anteriormente, tanto en Lamas (1912) como en el Plan de acción de la CNEF. No se trata de impedir que los "no aptos" se reproduzcan tal como postularía un eugenismo negativo, tampoco se alienta la reproducción de los "más aptos", más bien se trataría de una intervención sobre lo orgánico para ser "aptos", mejorar el organismo, o si se quiere, normalizar el cuerpo-organismo.

La novedad del siglo XIX fue según Foucault (2000) la aparición de una biología de tipo racista centrada en nociones evolucionistas y de degeneración. En definitiva tal como lo afirma el autor (2000: 80): "el Estado no es el instrumento de una raza contra otra...", al contrario el signo que adquiere es el de: “...protector de la integralidad, la superioridad y la pureza de la raza", "es" y "debe" serlo. Relación con el planteo foucaultiano tiene lo que propone José Pedro Barrán (1995) en el libro Medicina y Sociedad en el Uruguay del 900, el historiador pone de manifiesto cómo se estableció en Uruguay la relación entre "reforma social y eugenismo" (Barrán, 1995: 206). El concepto de eugenismo fue acuñado como estandarte por los médicos de la época, tornándose por momentos, más, una ideología “era convicción de los médicos de que su saber tenía el derecho y la obligación de regular la vida humana y modelar tanto el cuerpo del hombre como la vida de la espacie humana" (Barrán, 1995: 206). La eugenesia, teoría preocupada por la lucha contra la degeneración de la raza, fue un problema instigante para los médicos de la época que, encontraban en las leyes biológicas, la analogía de las leyes naturales, con la particularidad que el hombre a diferencia de la "naturaleza" podría guiar de manera inteligente dichas leyes, pudiendo combatir de este modo la degeneración, producto de las enfermedades y los vicios populares, tales como el alcoholismo, sífilis, tuberculosis y dolencias mentales (Barrán, 1995: 206). En el Plan de acción de la CNEF, al definir una Plaza de deportes versa que es el lugar en donde se guían de manera inteligente las conductas de los niños y jóvenes, se cultivan las buenas cualidades morales, y se propician actitudes sanas y saludables (CNEF, 1923). Contraponiendo a esto, el ambiente de los café, lugares con atmósferas 
viciadas, propicios para "el alcoholismo con sus devastadores efectos físicos y morales" (CNEF, 1923: 42).

En un trabajo de Platarrueda (2004), se aborda, a partir de los planteos de Stepan, la recepción que tuvo el eugenismo en los países latinoamericanos, identificando que:

La ideas neo-Lamarckianas ${ }^{17}$ justificaron la creencia de que el esfuerzo humano tenía significado, que los mejoramientos adquiridos a lo largo de la vida de un individuo podrían ser apropiados genéticamente, que el progreso podría ocurrir. De tal modo, la eugenesia empezó a estar vinculada a la obstetricia, las políticas de población, el bienestar del infante, la higiene mental y las campañas contra el alcoholismo, la tuberculosis y las enfermedades venéreas (es decir, contra los "venenos raciales"), entre otras muchas manifestaciones del intento de saneamiento y moralización de las naciones (Platarrueda, 2004:112).

El discurso de la eugenésia, tal como se puede leer en Platurrueda, no solo estuvo asociada a la idea de degeneración sino también de mejora biológica de la raza vinculada a una constante referencia a la moral. En un trabajo anterior, al analizar el discurso de Lamas ${ }^{18}$, encontré lo siguiente: “[Este autor] propone un plan de cultura corporal que sometió a juicio de las autoridades del cuerpo médico escolar de ese momento" (Caldeiro, 2017: 93), el mismo fue presentado el 4 de noviembre de 1911 en el Ateneo de Montevideo en una conferencia, publicada con patrocinio de la CNEF, titulada Educación física e intelectual conexas. Un plan de educación física infantil. En dicha publicación se refiere a la infancia y el juego, y expresaba que el niño en el juego "se somete a cierta disciplina voluntariamente", "le obliga a solidarizarse con los compañeros", "corrigen impulsos y ademanes groseros y antiestéticos"; por otra parte,

\footnotetext{
${ }^{17}$ La idea básica de la teoría de Lamarck era la siguiente: el entorno cambia las formas de vida luchan por adaptarse continuamente a las nuevas exigencias de su hábitat, estos esfuerzos modifican sus cuerpos físicamente, y estos cambios físicos son heredados por la descendencia. Es decir, que la evolución que proponía la teoría de Lamarck era un proceso que se sostiene en un concepto llamado herencia de las características adquiridas: los padres transmiten a los hijos los rasgos que adquieren a partir de cómo se relacionan con el entorno. Tomado de http://leucocitos17.blogspot.com.uy/2017/10/hipotesis-evolutivasde-lamarck.html

${ }^{18}$ Alejandro Lamas fue dentista, Profesor de Educación Física e ganador del primer concurso para formar docentes para el dictado de las primeras aulas "sistemáticas de 'gimnasia' y juegos a partir del año 1905". A comienzos de 1905 fue nominado profesor de Gimnasia de las escuelas de Montevideo. A partir del año 1908 se encargó del dictado de aulas de "gimnasia" en los institutos magistrales, tarea que desenvolvió por poco tiempo. En 1909 fue el principal conferencista de la $\mathbf{2}^{\mathbf{a}}$ Conferencia Pedagógica, Una lección de gimnasia. Su valor fisiológico, higiénico y pedagógico, en la que expuso sus ideas a partir de 16 lecciones de gimnasia que conformaban el programa propuesto por él para las escuelas (Dogliotti, 2012: 95-96). A su vez, escribió en 1903 el primer texto de Educación Física y Manual de Gimnasia Escolar.
} 
además de educar, el "juego metodizado recrea", "todos juegan y participan de la emotividad del juego, logrando cada cual su parte de alegría y beneficio fisiológico" (Lamas, 1912: 40). El discurso asocia ideas del orden de lo moral como pueden ser los comportamientos con los compañeros y la disciplina e ideas del orden de lo orgánico refiriéndose como mejoras fisiológicas. En la misma conferencia Lamas cita un "acuerdo", que surge del Congreso de Higiene Escolar que tuvo lugar en 1907, "la escuela es peligrosa si no garante al niño contra las enfermedades en incubación por deficiencias de higiene y si no favorece la evolución de su organismo por una educación física apropiada" (Lamas, 1911: 28). Se puede observar una preocupación por las condiciones higiénicas del ambiente en el cual permanece el cuerpo del niño, es necesario protegerlo de los peligros que lo amenazan y al mismo tiempo ofrecer estrategias para garantizar el desarrollo de su organismo.

La previsión de un fin catastrófico de la "raza" se dio paradojalmente la mano con su opuesta, la utopía de un perfeccionamiento físico, moral e intelectual indefinido del hombre que la medicina venía acariciando desde fines del siglo XVIII con Condorcet. Ambas convicciones partían de la misma base: la especie humana y la "raza" se construía con el esfuerzo de las generaciones sucesivas pero también podía destruirse o degenerarse por efecto de las enfermedades que atacaban esa cadena de la que la generación presente era solo un eslabón (Barrán, 1995: 207).

Esta perspectiva reclamaba acciones que pusieran al amparo la vida de la población, se proyectan en este sentido, las políticas de la vida.

\section{Acciones por una política de la vida}

La civilización y la industria moderna, han determinado, pues, lo que podríamos llamar una degeneración de la raza humana, y reclaman urgentemente actividades de diferentes clases como fuerzas compensatorias. (CNEF, 1923, p. 6).

El Plan de Acción de la CNEF fue aprobado en 1923 con el fin de ser desenvuelto en diferentes centros de enseñanza ${ }^{19}$, culturales y deportivos. Comienza presentando a modo de introducción y justificación un breve marco conceptual, en el cual se argumenta en favor de la importancia de la Educación Física en el combate a los efectos

\footnotetext{
${ }^{19}$ Comprendiendo las plazas de deportes, la Escuela Primaria, la universidad, la formación de profesores de Educación Física y el Patronato de delincuentes y menores.
} 
degradantes sobre el cuerpo, generados por la civilización. Los fundamentos utilizados devienen de las "ciencias modernas", responsables de un discurso vinculado a la educación neuromuscular y a la psicología moderna, las cuales

[consideran] a los músculos como órganos de expresión de los procesos eferentes. Más allá de sus funciones demostrables, cada cambio de atención, y de estado psíquico, generalmente acciona sobre ellos inconscientemente, modificando su tensión de una manera sutil, así que ellos no sólo podrían identificarse como órgano de la voluntad, sino también como del pensamiento y sentimiento. Las áreas motoras del cerebro, están íntimamente relacionadas y son considerablemente similares a las psíquicas y todavía no se ha comprobado que haya otro proceso que desarrolle más los centros cerebrales que la cultura muscular (CNEF, 1923, p. 2).

Como sostiene Dogliotti (2012) se produce una muscularización de la moral y una muscularización del cerebro. Esta idea de totalización muscular es un síntoma que puede darnos pistas para pensar nuevos emergentes en la Educación Física en las proximidades de la década del 20 del siglo XX, reedición de discursos respecto del cuerpo y de aparición de nuevas prácticas. Juegos, juegos deportivos, músculo, energización, carácter son conceptos que comienzan a ocupar lugar en los documentos de la época, y prácticas que se tornan poco a poco hegemónicas en el campo en cuestión. Al final del texto, en el punto referente a la Educación Neuro-muscular se afirma: "Los músculos son los vehículos del hábito, la obediencia, la imitación, del carácter y aún de costumbres y maneras" (CNEF, 1923: 3). Esta discursividad científica sobre la conducta humana y el músculo será la que oriente la producción de verdad sobre el cuerpo y la educación de los cuerpos en ese plan, para corregir y contrarrestar los efectos de la civilización, contribuyendo a la formación de "ciudadanos fuertes y saludables de espíritu, mente y cuerpo" (CNEF, 1923: 3). Los músculos son considerados órganos de la voluntad, por tanto una educación física centrada en el conocimiento del funcionamiento de estos órganos orientada por el faro de la moral permitiría el desenvolvimiento e incorporación de las "buenas costumbres y maneras". El cuerpo es reducido a organismo, al buen o mal funcionamiento de este. Los cambios en los "hábitos de vida" son la causa de las condiciones defectuosas del cuerpo del hombre moderno. Las transformaciones en la "industria", en los "métodos de trabajo" y la "vida sedentaria de las oficinas y de las escuelas" contribuyeron a "degenerar" el "vigor" (CNEF, 1923: 4) y las dimensiones de las estructuras del cuerpo. La civilización es presentada como una amenaza contra la cual es necesario luchar. Se concluye la 
primera parte del plan reafirmando que la civilización y la industria moderna fueron las responsables por la degeneración de la raza, por lo que se reclaman "prácticas de compensación" para este problema. En tal sentido es presentado un sistema de ejercicios y juegos que se desarrolla en el apartado "Sistemas y métodos". En este punto se presenta la forma de combatir los efectos acarreados por la modernidad y los antecedentes que diferentes pueblos adoptaron.

Considerando, como dijimos anteriormente, que la base epistémica de la Educación Física es constituida por las "ciencias biológicas y psicológicas", tiene sentido que los $\operatorname{objetivos}^{20}$ sean: "salud", "recreación física", "mejoramiento de la energía mental” y “carácter”. Como destacamos en páginas anteriores, Williams (2012: 113 - 114) dice que uno de los elementos centrales de la teoría darwinista social es que el carácter lo determina el entorno, algunos interpretaron, afirma el autor, la posibilidad de la reforma del carácter moral, tal fue el caso de Robert Owen, convencido que en un corto período de tiempo, modificando el entorno sería posible reformar el carácter moral de toda la población. El documento del Plan de acción, al desarrollar el cuarto objetivo, Carácter, versa:

El carácter es el resultado de la conformación o del ajuste del individuo a su medio ambiente. La naturaleza del carácter es determinada por la naturaleza del medio ambiente, en que se ha desarrollado aquella conformación. La educación física proporciona un medio ambiente, muy favorable para el cultivo del carácter [...] El campo de juego es una excelente escuela moral. Allí se aprenden prácticamente leyes, cuyos principios están basados en lo propios códigos sociales (CNEF, 1923: 14).

Al describirse en el Plan la población del país, se la anunciaba como descendiente de emigrantes europeos, principalmente de España e Italia, conformando un país relativamente joven

La raza indígena, que primitivamente habitaba estas tierras ha desaparecido totalmente, de modo que en la actualidad, es imposible dar con un indígena puro, pues sólo existen nativos descendientes de europeos de los diversos países que han poblado estas tierras. De modo que

\footnotetext{
${ }^{20}$ Según el Plan de Acción (CNEF, 1923), estos cuatro grandes objetivos deben ser seguidos en cualquier tipo de institución, ya fuera plaza de deportes, escuela, liceo, instituto correccional, cárcel, organización deportiva. Todas estas instituciones tenían educación física a cargo de la CNEF.
} 
nosotros heredamos las cualidades y características de los pueblos de los cuales descendemos (CNEF, 1923: 9).

Se aclara en el texto del Plan que, esa "herencia" a la que se hace referencia en la cita que pre sigue, no es un producto perfecto, sino que tiene "defectos" y "necesidades", y que se poseen los conocimientos de "los medios más eficaces para corregirlos y perfeccionarlos" (CNEF, 1923: 10). La Educación Física encuentra un lugar fecundo en este marco de posibilidad, acuñando la creencia que la herencia puede ser mejorada mediante una de educación del cuerpo del ciudadano uruguayo con objetivos sobre lo orgánico y con discursos totalizantes del cuerpo, como ya fue destacado por (Dogliotti, 2012), (Rodríguez, 2012): el hombre es reconocido por la Educación Física como un cuerpo orgánico y esta visión cubre todas aquellas prácticas desenvueltas a su alcance.

El cuerpo del niño, indica el Plan, está "expuesto a muchos peligros que conspiran contra su organismo", entonces

si se defiende bien de los numerosos enemigos que lo atacan $[\ldots]$ demuestra estar preparado para pasar el próximo período de su vida; el último tramo de la infancia que lo une con la adolescencia (CNEF, 1923: 87).

Ante la presencia de amenazas constantes al organismo en el período de la infancia, la Educación Física encuentra la justificación para su acción, el discurso y las prácticas producen la idea del cuidado de la raza. Se procede identificando la presencia de un peligro biológico pero también psicológico, "Desde el punto de vista psicológico, el niño en este período se asemeja a un indio incivilizado" (CNEF, 1923: 87) y por ello se justifica la intervención. Diagnóstico y receta. Como los vanguardistas en la adopción de diferentes procedimientos, con los que afrontaron las problemáticas que atentaban "contra la salud de sus nuevas generaciones", se destaca en el Plan, a los países desarrollados -Estados Unidos, Suecia, Alemania, Francia, Suiza-. Se convoca, entonces desde el Plan, apoyar esa "obra nacional" ya que aportaría "grandes beneficios a nuestras generaciones nuevas y evitaría en lo futuro al Estado grandes gastos, favoreciendo al mismo tiempo el engrandecimiento nacional" (CNEF, 1923: 91). Y se dice más: durante los primeros 10 años de vida del niño, "el objetivo principal es el crecimiento y desarrollo perfectos de su organismo"; es en el marco de una discursividad que teje los conceptos de organismo, raza, normalidad, desarrollo, degeneración que se justifica y se "reclama un Plan de Educación Física". 


\subsection{Un plan de Educación Física escolar}

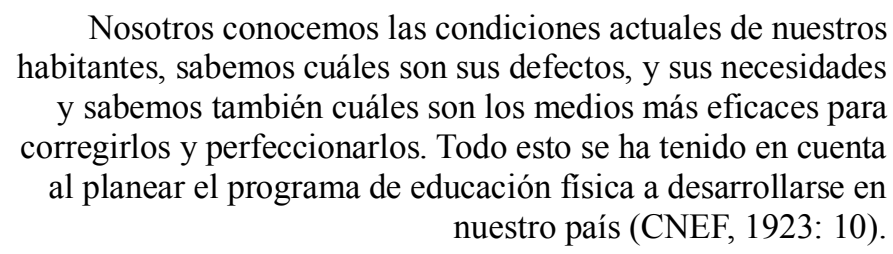

El apartado destinado a la Educación Física en las escuelas públicas describe al niño en edad escolar -de los 6 a los 12 o 13 años- como un organismo que se encuentra en desarrollo,

En esta época el niño demanda mucha comida sana y buena, largas horas al aire libre y prolongado y profundo sueño. Su organismo ahorra energías, que tendrá que emplear en la gran metamorfosis que se acerca, que lo convertirá de un niño en un adulto joven, o adolescente. (CNEF, 1923: 87).

Los objetivos que debería tener un plan de Educación Física escolar se centran en corregir y mejorar aquellos aspectos que ponen en riesgo el desarrollo "normal", por ejemplo, la "corrección de las malas tendencias de la vida escolar" (CNEF, 1930: 19). El programa a seguir es el de los "ejercicios llamados de descanso o compensación", a través de los cuales se consiguen: "buenas posturas", y "disciplina, por medio de comandos a los cuales haya que responder de inmediato con energía y vivacidad", como asimismo "salud" (CNEF, 1930: 19 - 20). El tercer objetivo propuesto trata del “mejoramiento de las condiciones saludables e higiénicas de la escuela [...], [lo que sería posible mediante] confección de la ficha médico-sanitaria-antropométrica. [...] Este examen sirve también para establecer grupos homogéneos para la práctica de ejercicios y juegos" (CNEF, 1930: 21). Una vez hecho el examen se presta apoyo a las familias que requieran de atención especial, ya sea de orden físico, higiénico o moral (CNEF, 1930: 21). Por último, a aquellos niños o niñas a los cuales les fueran detectadas "serias debilidades orgánicas", se sugería en términos de "deber", que fueran exonerados de la práctica de ejercicios generales, y se derivaran a trabajos correctivos, siendo examinados periódicamente (CNEF, 1930: 21 - 22). Para poder jugar se exige superar el examen, es decir: a partir de mediciones sanitarias y antropométricas, y de la noción de degeneración, se recorta una zona de enfermedad, de peligro social sustentada en el saber médico, la cual se aísla, derivándola a trabajos de regeneración, al tiempo que se conforman grupos homogéneos para los juegos. Se marca un corte importante 
respecto del juego, no se trata de prohibirlo o reprimirlo sino de una modificación de sus sentidos. El nuevo juego exigía, para poder jugarse, estar apto físicamente y desde el punto de vista higiénico y moral, es decir, pertenecer a la buena raza, condición primera para poder jugar los deportes. Una cuarta estrategia vinculada a la enseñanza teórica y práctica de la higiene es sugerida en el documento. Se recomienda la construcción de un manual y que el maestro de aula dedique 10 o 15 minutos a hablar sobre su contenido (higiene, aseo individual) (CNEF, 1930: 22). Finalmente, se indica en el plan escolar actividades e iniciativas especiales como estrategias que auxiliarían el proceso de higienización de los cuerpos emprendido por la Educación Física. Tales iniciativas eran: la liga deportiva escolar, los clubes de salud y el examen de triple postura, del cual los estudiantes podrían sacar "saludables enseñanzas". Las finalidades de estos dispositivos eran estimular la práctica de hábitos higiénicos, recomendándose la organización de competencias al interior de la escuela (esto último tanto para la liga deportiva escolar como para los clubes de salud).

\subsection{Campamentos escolares: bio-dispositivo al aire libre}

Los campamentos de escolares fueron una estrategia pedagógica bajo la dirección de la $\mathrm{CNEF}$, organizados en época de vacaciones, "campamentos infantiles, a los cuales concurren niños ${ }^{21}$ de las escuelas públicas" (CNEF, 1930: 32).

Los niños que concurren a esos campamentos no tienen que gastar ni un solo centésimo, y son elegidos en las escuelas ubicadas en barrios densa población. Deben proceder de familias de modesta condición social, y no ser enfermos, sino débiles o que necesiten hacer vida de campo o de playa. La selección de niños es hecha por el personal docente de la escuela, el cuerpo Médico Escolar y la Oficina Médica de la Comisión Nacional (CNEF, 1930: 32. El destacado es nuestro).

El dispositivo "campamento" estaba orientado a los niños de modestos recursos que presentaran, según el criterio médico, debilidades, que se regenerarían con una adecuada vida de campo y playa. A su vez eran seleccionados de zonas con densidad de población, este último aspecto deja trasparentar el miedo a la muchedumbre, que se

\footnotetext{
${ }^{21}$ Creemos que a estos campamentos concurrían solamente varones, ya que en ningún momento se hace referencia a las niñas, siempre se habla de niños, cuestión que podría responder a los modos del lenguaje; no se distinguen actividades por sexo cosa que era común en esa época, y tampoco se puede ver en las imágenes presencia de niñas.
} 
mencionaba más arriba en el texto, propio del eugenismo de los Estados Unidos de Norte América. El recorte de la zona de riesgo deja por fuera a los niños enfermos. La discriminación realizada selecciona barrios en los que la población se acumula, la condición social modesta característica de los barrios de población densa eran los que se tornaban una amenaza biológica y por lo tanto, una política implementada a tiempo evitaría la enfermedad, así como el contagio y por lo tanto el fortalecimiento de la raza. Los campamentos se tornaron una estrategia biopolítica con el fin del mejoramiento físico de los niños seleccionados.

Los mismos se estructuraban con una duración, de entre 15 y 21 días, y siempre que fuera posible se sugería cumplir con la máxima duración estipulada. Los niños en el campamento eran divididos en grupos de 12 a 14 por cabaña con un responsable a cargo, "instructor" quien se encargará del cuidado y vigilancia permanente, pero no solo, sino que también los aconsejaría e instruiría en temas variados (CNEF, 1930: 33). Este tipo de procedimiento típico de las disciplinas se asociaba con un programa de actividades diario, en el que se marca en detalle horarios y actividades a realizar,

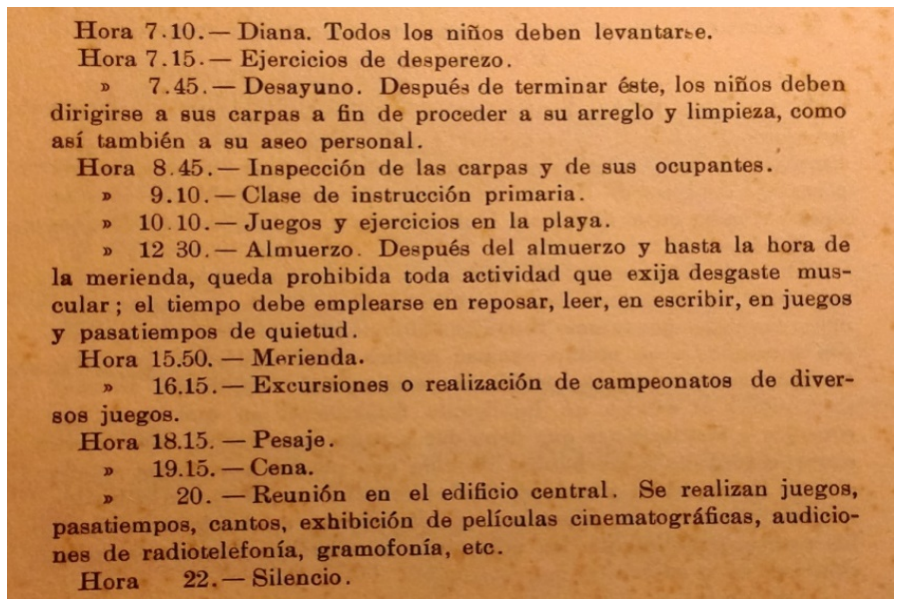

Imagen 1. Fuente: CNEF, 1930.

Como se puede apreciar en el programa, 15 horas del día están puntillosamente organizadas y prescriptas por actividades de tipo higiénico desde los ejercicios matinales, pasando por el aseo personal, la limpieza de las cabañas, hasta las inspecciones matinales tanto de las cabañas como de sus ocupantes. Esta rutina diaria que se resolvía en secuencias de descanso, ejercicios y alimentación acompañadas de prescripciones de tipo higiénicas durante el tiempo de campamento pretendía la incorporación de hábitos saludables por parte de los acampantes, hábitos que evitarían 
la degeneración de la vida y a la vez la regeneración delas condiciones defectuosas por las cuales habían sido seleccionados los niños.

En las imágenes del $\mathrm{N}^{\circ} 2$ a la 5 que se presentan a continuación se pueden apreciar las diferentes actividades realizadas en los campamentos que, constituían la rutina diaria a la que hacíamos referencia anteriormente. Las imágenes $\mathrm{N}^{\circ} 2,3$ y 4 atestiguan el lugar que ocupó la disciplina, los cuerpos ordenados distribuidos equitativamente en el espacio, la postura erguida, la mirada al frente, la secuencia del gesto ejecutada al unísono. Así mismo muestran el lugar de la higiene en las prácticas campamentiles, tanto los ejercicios matinales de gimnasia sueca, representativos de una práctica con movimientos limitados al lugar, centrada fundamentalmente en lo correctivo y lo terapéutico, que busca la corrección de las posturas viciadas y el reforzamiento de los grupos musculares debilitados, se repetía cada mañana con el objetivo de incorporarse como hábito. Tanto una correcta limpieza de dientes, como el orden de la cama eran reflejo de prácticas higiénicas que se buscaron inculcar mediante estos dispositivos, expresión del cuidado de sí.

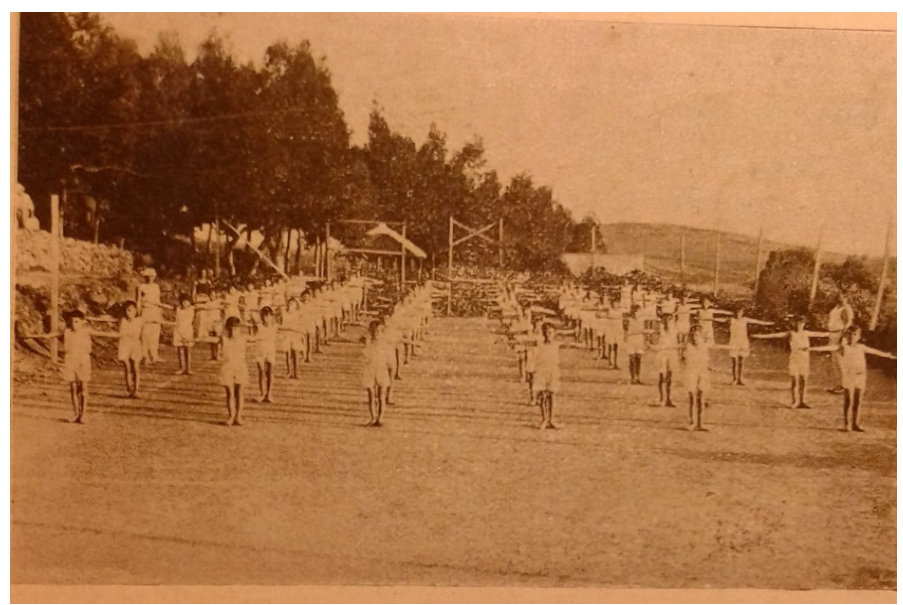

Imagen 2. Ejercicios, por la mañana, después de levantarse. Fuente: CNEF, 1930. 


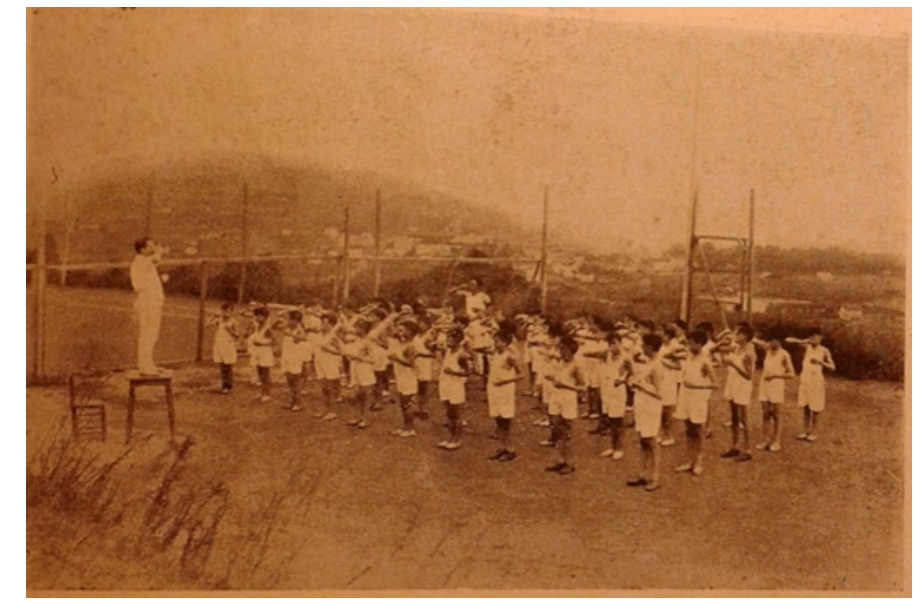

Imagen 3. Una lección de higiene bucal. Fuente: CNEF, 1930.

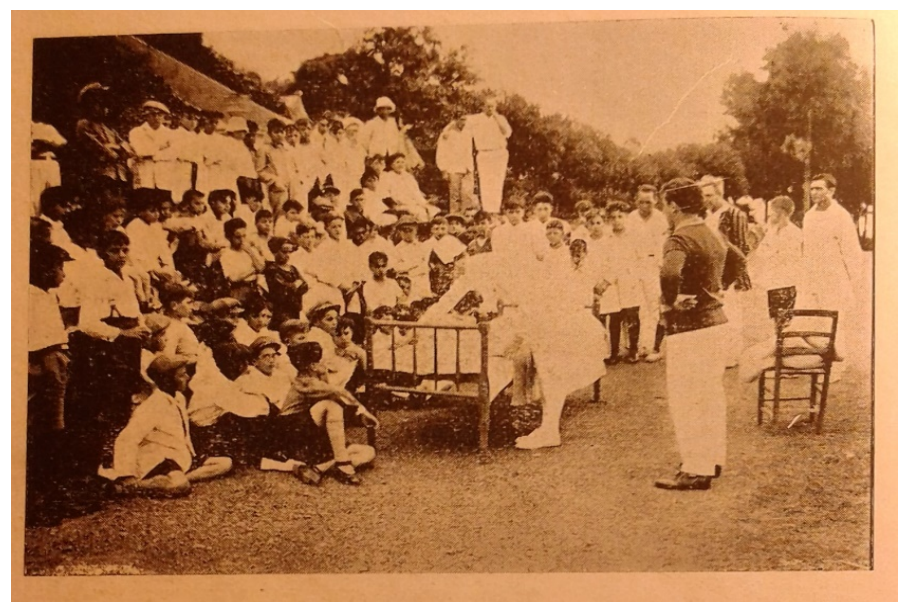

Imagen 4. Lección colectiva sobre la manera de armar la cama. Fuente: CNEF, 1930.

Este dispositivo deja traslucir la manera cómo se recepcionó el discurso vinculado a la eugenesia a través de prácticas higiénicas por parte de la Educación Física, del mismo modo se identifican prácticas asociadas al cuidado y mejoramiento del organismo, se advierte la creencia que el mejoramiento producto del esfuerzo y el trabajo sobre el cuerpo adquiridos a lo largo de la vida de un individuo podrían ser apropiados genéticamente, que el progreso podría ocurrir, lo que justifica las acciones vinculada a las políticas de población y del bienestar del infante. 


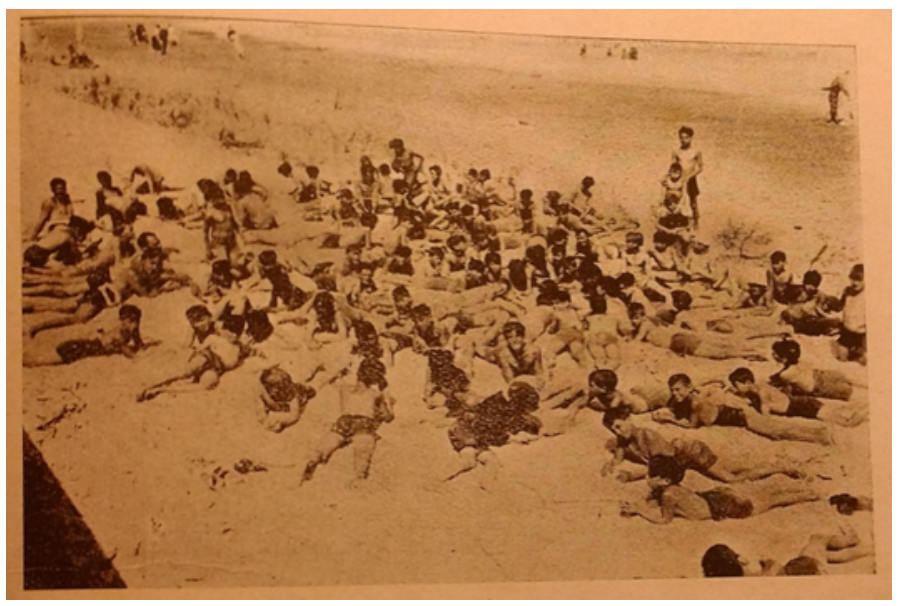

Imagen 5. Baño de sol en la playa. Fuente: CNEF, 1930.

Baños de sol: fuente de "vida". La playa también fue en un lugar propicio para el higienismo. El aire libre, el mar y el sol eran reconocidos como fuentes regenerativas de las condiciones degeneradas. Correr al aire libre permitiría el intercambio gaseoso purificante de los pulmones, el mar, purificador del alma y la epidermis bronceada daba cuenta de un cutis lleno de vida.

Tal como es señalado por Nancy Stepan (1991), la higiene se tornó un método para mejorar la raza. El enfoque higiénico rector de la Educación Física de la época promovió prácticas al aire libre como estrategias para combatir la degeneración del cuerpo, ya sea mediante los baños de sol, los ejercicios propios de la disciplina o los juegos, tal como se puede ver en las imágenes 5, 6 y 7 respectivamente.

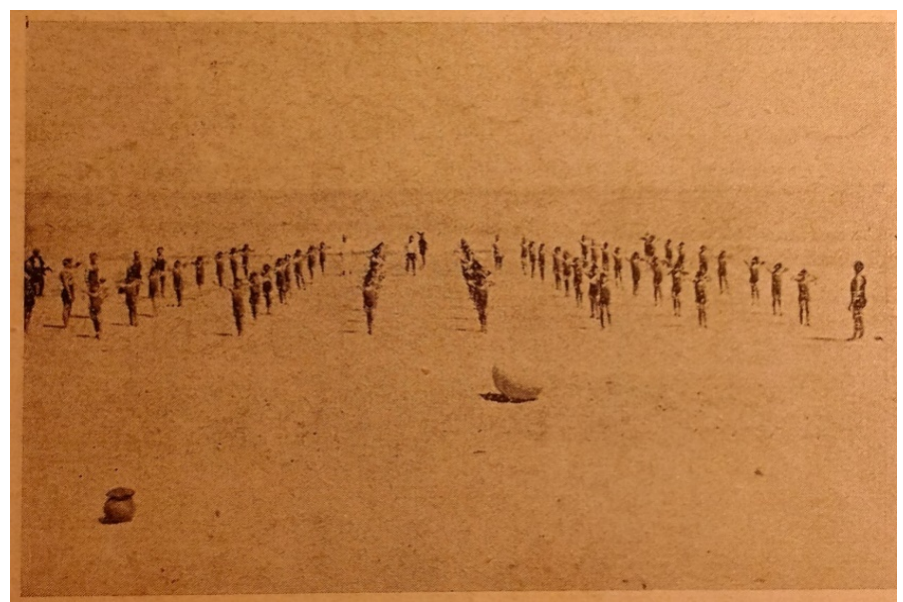

Imagen 6. Ejercicios en la playa. Fuente: CNEF, 1930. 


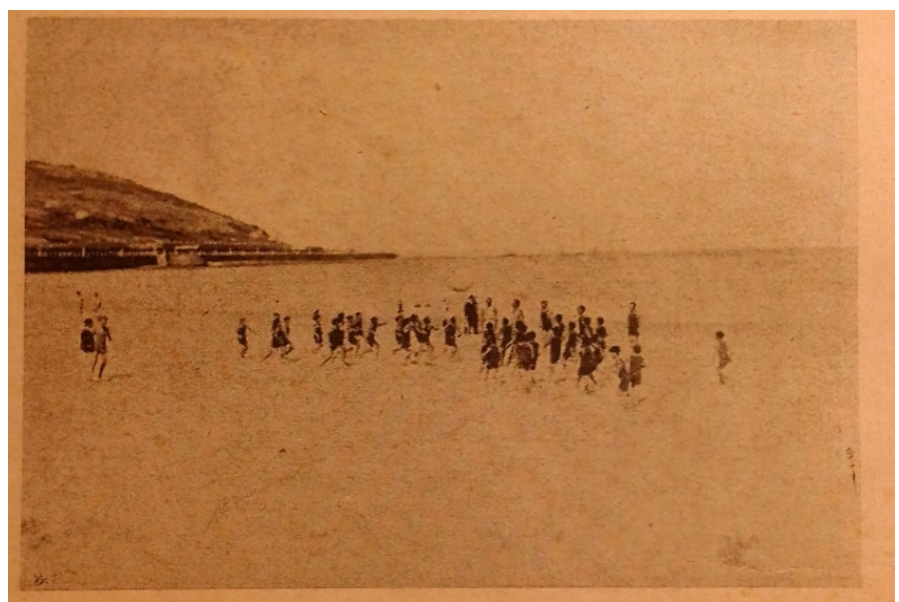

Imagen 7. Juegos en la playa. Fuente: CNEF, 1930.

Al hacer referencia a los resultados de estos campamentos, el documento de 1930, los valoraba como óptimos, y por momentos parece depositárseles exagerados beneficios. Se le adjudica al dispositivo "campamento" un poder de renovación cuasi omnipotente, veamos cómo se describen los efectos de los campamentos:

Los niños salen de sus hogares con un aspecto enfermizo, la tez pálida, las mucosas descoloridas, la mirada inexpresiva, la actitud agobiadora, etc, y regresan a los mismos rebosantes de salud, con el cutis bronceado por el sol, la mirada viva, las mucosas enrojecidas, con mayor peso, llenos de alegría, enriquecidos en experiencias y buenos hábitos y con un enorme bagaje de nuevos conocimientos adquiridos en la vida de campamento (CNEF, 1930: $33)$.

La cuestión de la preocupación por la degeneración de la población se hace explícita en la cita anterior. Los conocimientos a los que se hace referencia, son los hábitos y prácticas higiénicas aprendidas como resultado de la experiencia del campamento. Los niños seleccionados para concurrir al campamento se caracterizaban por presentar ciertas debilidades físicas detectadas por los médicos, las cuales serían modificadas en los campamentos escolares. Como ya había sido declarado por Varela ${ }^{22}$ en 1876 "...el color cadavérico, el pecho hundido y las naturalezas débiles y enfermizas que tan a

22 "José Pedro Varela (1845-1879) ha sido considerado el Padre de la escuela uruguaya "laica, gratuita y obligatoria", también llamado el Reformador. Varela es autor del Proyecto de Ley General de Educación Común. La Legislación Escolar, publicada en 1876, fue motivada por dicho proyecto de ley. La obligatoriedad de la enseñanza primaria se concreta en el Decreto Ley de Educación Común del 24 de agosto de 1877. En La Educación del Pueblo (1874) y La Legislación Escolar (1876) se condensa el ideario pedagógico vareliano, ideario que se constituirá como matriz identitaria de la educación pública uruguaya hasta nuestros días" (Rodríguez, 2012: 60). 
menudo se encuentran entre nosotros" se atribuían a la falta de ejercicios físicos que tan buenos resultados provocaban en las poblaciones anglo-sajonas y alemanas, estas se caracterizaban -según la descripción de Varela- por “...la coloración viva, la elevada estatura, el pecho levantado, y el vigor y la robustez..." (Varela 1876 apud Dogliotti, 2012: 65 - 66). Es interesante ver la continuidad del discurso vareliano, que se mantiene durante las primeras tres décadas del siglo XX. El cuerpo inferior físicamente representaba un problema a ser atacado para lograr una buena raza, el campamento permitía una transformación total, los niños volvían: “...rebosantes de salud, con el cutis bronceado [...], la mirada viva, las mucosas enrojecidas, con mayor peso, llenos de alegría, enriquecidos en experiencias y buenos hábitos...” (CNEF, 1930: 33). El problema de los cuerpos, es decir la preocupación por la degeneración de la raza se vuelve foco de políticas educativas, o mejor podríamos decir se torna una bio-política que tomó en sus manos la población infantil.

\section{A modo de cierre}

El siglo XX fue un momento de emergencia de la teoría eugenista tal como lo sostiene Stepan (1991), y así mismo Barrán (1995) plantea que en las primeras cuatro décadas del siglo XX en Uruguay "el eugenismo o sus ideas claves" encontraron un lugar fecundo para su difusión e influencia. Este trabajo no tuvo como preocupación central responder la preguntarse qué es la eugenesia, ni tampoco, si se cumplieron al pie de la letra los principios de esta teoría, sino más bien, el interés fue identificar la recepción de ese discurso en el marco de la Educación Física con explicitas preocupaciones higiénicas focalizada en la infancia y de qué forma operó en las prácticas, en un Uruguay marcado por una época de reformas sociales y producción del ciudadano.

La preocupación por la Infancia fue una cuestión que ocupó a médicos y pedagogos de la época, desde finales del siglo XIX y comienzos del siglo XX. Una creencia explícita en la posibilidad de influir en las mejoras orgánicas del cuerpo infantil, está expresado en las palabras de Lamas (1912) al afirmar que "la puericultura y fisicultura son cosa de estos días [...], y que exigen una serie de conocimientos que sólo contemporáneamente se hacen y deben hacerse en adelante por quienes pretendan educar organismos infantiles" (Lamas, 1912: 36). La puericultura, se presenta como la protección a la infancia centrada en la educación higiénica como forma de salvaguardar una herencia 
genética modificada por el ambiente, esta cuestión coloca un trazo particular a la recepción de la teoría impulsada por Galton que defendía la influencia decisiva de la herencia. Diferentes dispositivos fueron montados para asegurar la vida, desplegando estrategias diversas haciendo foco en la producción de la Infancia, con el fin de asegurar la raza. La Educación Física, en tanto política del Estado uruguayo como forma de educar los cuerpos de los ciudadanos, tuvo aparentemente una recepción del discurso eugenésico. Las prácticas desenvueltas en su seno se impregnaron de esa teoría que funcionó por momentos como una ideología del cuerpo. La noción de degeneración, característica del discurso racista, permite aislar, recortar una zona de peligro social al mismo tiempo que se le da un estatuto de enfermedad, la cual, debe ser combatida, sometiéndola a "fuerzas compensatorias", "actividades" que contrasten cualquier deterioro orgánico. La CNEF implementó diferentes estrategias para combatir los efectos negativos de la civilización, adoptando, "sistemas de ejercicios y juegos" (CNEF, 1923: 6), implementados por pueblos avanzados (Estados Unidos, Alemania, Francia, Suecia, entre otros) quienes "obtuvieron buenos resultados" (CNEF, 1923). En este mismo marco discursivo la práctica de campamento se tornó otra estrategia de vida higiénica, quedando inscripta en los principios de mejora de la raza. Otro indicio que da cuenta de la presencia y manera en como operó el discurso de la eugenesia, se puede ver en los exámenes médicos del período "ficha médico-sanitaria-antropométrica" implementada en las escuelas siendo sus efectos la individualización biológica, que permitió inscribir en serie a cada sujeto a partir de la medición de las condiciones corporales y orgánicas, habilitando a quienes pasaran el examen, es decir, los aptos físicamente a poder jugar. Un último aspecto que demuestra la influencia del eugenismo, son las argumentaciones psicologistas, tal como aparece en el plan de acción de 1923, cuando afirma que la Educación Física es una ciencia "basada en las ciencias biológicas y psicológica" (CNEF, 1923: 9).

\section{Referencias bibliográficas}

Ariès, P. (1987). El descubrimiento de la infancia. En: El niño y la vida familiar en el antiguo régimen. Madrid: Taurus.

Barrán, J. (1995). Medicina y sociedad en el Uruguay del Novecientos. La invención del cuerpo. Montevideo: Banda Oriental, 1995. 
Barrán, J. (1999). Biología, Medicina y Eugenesia en Uruguay. Disponible en: http://asclepio.revistas.csic.es/index.php/asclepio

Barrán, J. (2009). Historia de la sensibilidad en el Uruguay. Montevideo: Banda Oriental.

Caetano, G. (2011). La República Batllista. Montevideo: Banda Oriental.

Caldeiro, M. (2017). O corpo em jogo: a emergência do discurso sobre o jogo no campo da Educação Física no início do século XX no Uruguai. Disertación de maestría PPGE -UFSC.

COMISIÓN NACIONAL DE EDUCACIÓN FÍSICA (CNEF). (1923). Plan de Acción de la CNEF y Conclusiones que se derivan del mismo. Presentado por su Director Técnico Sr. Julio J. Rodríguez. Montevideo: CNEF.

COMISIÓN NACIONAL DE EDUCACIÓN FÍSICA (CNEF). (1930). Educación Física en el Uruguay, por Julio J. Rodríguez, Director Técnico General de la CNEF. In: Boletín del Instituto Internacional Americano de Protección a la Infancia. Montevideo: Comisión Nacional del Centenario 1830-1930.

Dogliotti, P. (2012). Cuerpo y currículum: discursividades en torno a la formación de docentes de educación física en Uruguay (1874-1948). Tesis de Maestría en Enseñaza Universitaria del Programa de Especialización y Maestría en Enseñanza Universitaria, Área Social y CSE, UdelaR, Montevideo.

Foucault, M. (2000). Defender la sociedad. Buenos Aires: Fondo de Cultura Económica.

Lamas, A. (1912) Educación física e intelectual conexas. Un plan de educación física infantil. Montevideo: Barreiro y Ramos.

Nahum, B. (2011). La época batllista. 1905 - 1929. Montevideo: Banda Oriental

Platarrueda, C. (2004). Usos tempranos de la antropología en la retórica eugenésica. Colombia. Cuadernos de los Seminarios. Disponible en: ttp://www.bdigital.unal.edu.co/1265/9/08CAPI07.pdf

Rodríguez, R. (2012). Saber del cuerpo: una exploración entre normalismo y universidad en ocasión de la educación física (Uruguay, 1876-1939). Tesis de 
Maestría en Enseñaza Universitaria del Programa de Especialización y Maestría en Enseñanza Universitaria, Área Social y CSE, UdelaR, Montevideo.

Stepan, N. (1991), The hour of eugenics: race, gender and nation in Latin America, New York, Cornell University Press.

Varela, J. (1876) La Legislación Escolar. Tomo I. Montevideo: Biblioteca Artigas. Colección de Clásicos Uruguayos. Vol. 51. Obras Pedagógicas, Montevideo, 1964.

Williams, R. (2012). Darwinismo social en Cultura y Materialismo. BsAs: la marca. 